\title{
ARTICLES
}

\section{Symplectic quaternion scheme for biophysical molecular dynamics}

\author{
T. F. Miller III, M. Eleftheriou, P. Pattnaik, A. Ndirango, and D. Newns \\ T. J. Watson Research Center, Yorktown Heights, New York 10598 \\ G. J. Martyna ${ }^{\text {a) }}$ \\ T. J. Watson Research Center, Yorktown Heights, New York 10598 and Department of Chemistry, \\ Indiana University, Bloomington, Indiana 47405-4001
}

(Received 11 January 2002; accepted 5 March 2002)

\begin{abstract}
Massively parallel biophysical molecular dynamics simulations, coupled with efficient methods, promise to open biologically significant time scales for study. In order to promote efficient fine-grained parallel algorithms with low communication overhead, the fast degrees of freedom in these complex systems can be divided into sets of rigid bodies. Here, a novel Hamiltonian form of a minimal, nonsingular representation of rigid body rotations, the unit quaternion, is derived, and a corresponding reversible, symplectic integrator is presented. The novel technique performs very well on both model and biophysical problems in accord with a formal theoretical analysis given within, which gives an explicit condition for an integrator to possess a conserved quantity, an explicit expression for the conserved quantity of a symplectic integrator, the latter following and in accord with Calvo and Sanz-Sarna, Numerical Hamiltonian Problems (1994), and extension of the explicit expression to general systems with a flat phase space. (c) 2002 American Institute of Physics. [DOI: 10.1063/1.1473654]
\end{abstract}

\section{INTRODUCTION}

In the last year, the human genome project has been largely completed, and the primary structures of the large majority of all human proteins are, now, available for study. ${ }^{1}$ This presents a unique opportunity for the field of computational biology to make contributions in the determination of the three-dimensional structures of new proteins, the discovery of protein substrates and inhibitors, and the generation of the mechanism by which proteins chemically modify molecules. However, computational methods and computer architectures of unprecedented speed, efficiency, and accuracy must be developed in order to process the vast quantities of data produced by the genome project.

Recently, IBM has announced a next-generation, massively parallel, supercomputer that will be employed to perform biophysical molecular dynamics (MD) simulation studies. ${ }^{2}$ This new advance in computational power will, in principle, allow long time scales to be sampled and new biological insights to be obtained. Unfortunately, current multiple time step MD integration methods, ${ }^{3,4}$ while symplectic and, thus, stable at long times, ${ }^{5}$ require large numbers of computationally inexpensive force evaluations to be performed. These operations limit the efficiency of the finegrained parallel decompositions employed on massively parallel supercomputers due to communication overhead as opposed to their negligible cost to actually compute.

The fast degrees of freedom in a large biomolecule and the solvent can be removed using holonomic constraints,

${ }^{a)}$ Electronic mail: gmartyna@indiana.edu thus, creating a dynamical system of coupled sets of rigid bodies with lower communication overhead. However, existing methods designed to treat sets of rigid bodies are either (a) solved iteratively and are, hence, not reversible ${ }^{6,7}$ and cannot be used in hybrid Monte Carlo (HMC) calculations; (b) are not symplectic and, hence, not stable at long times; ${ }^{8,9}$ or (c) introduce many extra parameters that must themselves be constrained and, hence, go beyond the four parameters required to define a nonsingular mapping of rigid body rotations. ${ }^{10,11}$ Finally, using modern methods, phase space volume preserving but nonsymplectic integrators have been developed ${ }^{12}$ but theoretical tools to formally assess the utility of this and similar approaches have been lacking. (Note, a symplectic integrator both possesses a time-step-dependent energy, which is invariant along the trajectory produced by the integrator, and is phase space volume preserving. A phase space volume preserving integrator need not be symplectic. Furthermore, neither phase space volume preservation nor the symplectic property quantity, guarantee that an integrator will possess time reversal symmetry.)

In this paper, the equations of motion for rigid bodies in terms of the unit quaternion, ${ }^{13}$ the minimal parameter set most commonly used in MD simulation as first proposed by Evans, ${ }^{8,9}$ are presented. A novel Hamiltonian or symplectic form of the equations is then derived and expressed as a sum over permutations. The new form, denoted NO_SQUISH (novel symplectic quaternIon scheme), allows a symplectic reversible integrator to be formulated, naturally, via the Trotter-Suzuki operator splitting approach., ${ }^{3,14}$ Next, a general explanation of the properties of numerical integrators is provided (symplectic, phase space volume preserving, time 
reversible, etc.). This is followed by a formal development that relates the existence of conserved quantities in the approximate integrators constructed using the Trotter-Suzuki approach to a simple recursion relation valid for both nonsymplectic and symplectic integrators. A general expression for the conserved quantities of symplectic integrator derived via the Trotter-Suzuki approach is given followed by a new extension to general systems with a flat phase space (defined within). Both the theory and NO_SQUISH are tested using comparisons to a reversible phase space volume preserving but nonsymplectic integration scheme for the unit quaternion equations, ${ }^{12}$ and the SHAKE/RATTLE method, ${ }^{6,7}$ which is neither reversible nor symplectic unless iterated to full convergence, ${ }^{15}$ on model and biophysically important problems.

\section{METHODS}

In this section, the novel rigid body techniques are developed. First, the standard equations for rigid body motion are expressed in terms of the unit quaternion. The equations are then recast in Hamiltonian form which is further reduced to a sum over permutation matrices using quaternion algebra. In order to understand better the properties of numerical integrators, a general discussion of the concepts such as time reversal symmetry and the symplectic property is given fol- lowed by a new treatment that expresses the condition for a conserved quantity to exist in a symplectic or nonsymplectic integrator in the form of a recursion relation. A precise expression for the conserved quantity of a symplectic integrator developed using the Trotter-Suzuki approach, is then derived and an extension to general systems with a flat phase space given. Last, the new symplectic integrator for the Hamiltonian form of the equations of motion is presented and the derivation of an older phase space volume preserving but nonsymplectic integrator is reviewed. ${ }^{12}$

\section{A. Equations of motion}

In the classical mechanics of rigid body motion, the unit quaternion, $\left\{q_{0}, q_{1}, q_{2}, q_{3}\right\}$ with $\Sigma_{i} q_{i}^{2}=1$, is introduced in order to generate a minimal, nonsingular, representation of the rotation matrix from a space-fixed (denoted " $s$ ") to a body-fixed coordinate system (denoted " $b$ ")

$$
\mathbf{r}^{(b)}=\mathbf{A}(\mathbf{q}) \mathbf{r}^{(s)}, \quad \mathbf{r}^{(s)}=\mathbf{A}^{T}(\mathbf{q}) \mathbf{r}^{(b)},
$$

in which the moment of inertia tensor $\mathbf{I}$

$$
\mathbf{I}_{\alpha \beta}=\sum_{k=1}^{N} m_{k}\left(\left|\mathbf{r}_{k}\right|^{2} \delta_{\alpha \beta}-\mathbf{r}_{k_{\alpha}} \mathbf{r}_{k_{\beta}}\right)
$$

is diagonal. ${ }^{13}$ Here,

$$
\mathbf{A}(\mathbf{q})=\left(\begin{array}{ccc}
q_{0}^{2}+q_{1}^{2}-q_{2}^{2}-q_{3}^{2} & 2\left(q_{1} q_{2}+q_{0} q_{3}\right) & 2\left(q_{1} q_{3}-q_{0} q_{2}\right) \\
2\left(q_{1} q_{2}-q_{0} q_{3}\right) & q_{0}^{2}-q_{1}^{2}+q_{2}^{2}-q_{3}^{2} & 2\left(q_{2} q_{3}+q_{0} q_{1}\right) \\
2\left(q_{1} q_{3}+q_{0} q_{2}\right) & 2\left(q_{2} q_{3}-q_{0} q_{1}\right) & q_{0}^{2}-q_{1}^{2}-q_{2}^{2}+q_{3}^{2}
\end{array}\right)
$$

and

$$
\begin{aligned}
& q_{0}=\cos \left(\frac{\theta}{2}\right) \cos \left(\frac{\phi+\psi}{2}\right), \quad q_{1}=\sin \left(\frac{\theta}{2}\right) \cos \left(\frac{\phi-\psi}{2}\right), \\
& q_{2}=\sin \left(\frac{\theta}{2}\right) \sin \left(\frac{\phi-\psi}{2}\right), \quad q_{3}=\cos \left(\frac{\theta}{2}\right) \sin \left(\frac{\phi+\psi}{2}\right),
\end{aligned}
$$

where $\theta, \phi$, and $\psi$ are the three Euler angles in the standard convention $^{13}$ and a body consisting of $N$ point particles at position $\mathbf{r}_{k}=\left\{\mathbf{r}_{k_{1}}, \mathbf{r}_{k_{2}} \mathbf{r}_{k_{3}}\right\}=\left\{x_{k}, y_{k}, z_{k}\right\}$, with mass $m_{k}$ assumed for convenience in the definition of the moment of inertia tensor (see Fig. 1) and the center of mass at the origin. Introducing the body-fixed angular velocities $\omega_{x}, \omega_{y}$, and $\omega_{z}$, the rigid body equations of motion can be written as,

$$
\begin{aligned}
& \dot{\mathbf{q}}=\frac{1}{2} \mathbf{S}(\mathbf{q}) \omega^{(4)}, \\
& \dot{\omega}_{x}=\frac{\tau_{x}}{I_{x x}}+\frac{\left(I_{y y}-I_{z z}\right)}{I_{x x}} \omega_{y} \omega_{z}, \\
& \dot{\omega}_{y}=\frac{\tau_{y}}{I_{y y}}+\frac{\left(I_{z z}-I_{x x}\right)}{I_{y y}} \omega_{z} \omega_{x}, \\
& \dot{\omega}_{z}=\frac{\tau_{z}}{I_{z z}}+\frac{\left(I_{x x}-I_{y y}\right)}{I_{z z}} \omega_{x} \omega_{y},
\end{aligned}
$$

where $\omega^{(4)}=\left(0, \omega_{x}, \omega_{y}, \omega_{z}\right)$,

$$
\mathbf{S}(\mathbf{q})=\left(\begin{array}{cccc}
q_{0} & -q_{1} & -q_{2} & -q_{3} \\
q_{1} & q_{0} & -q_{3} & q_{2} \\
q_{2} & q_{3} & q_{0} & -q_{1} \\
q_{3} & -q_{2} & q_{1} & q_{0}
\end{array}\right),
$$

$\left[\mathbf{S}(\mathbf{q}) \mathbf{S}^{T}(\mathbf{q})\right]_{\alpha \beta}=|q|^{2} \delta_{\alpha \beta}=\delta_{\alpha \beta}, \quad \tau$ are the torques in the body-fixed frame, and $I_{x x}, I_{y y}$, and $I_{z z}$ are the diagonal elements of the moment of inertia tensor (note, again, I is diagonal in the body fixed frame). Note, the equations embody the time derivative of the constraint, $\Sigma_{i} q_{i} \dot{q}_{i}=0$, via the first element of the vector $\omega^{(4)}=2 \mathbf{S}^{T}(\mathbf{q}) \dot{\mathbf{q}}$. The torques can be written in the form

$$
\tau^{(4)}=-\frac{1}{2} \mathbf{S}^{T}(\mathbf{q}) \nabla_{q} \phi(\mathbf{q})+\tau_{\text {int }}^{(4)},
$$

where $\phi(q)$ is the potential energy and $\tau_{\text {int }}^{(4)}$ is the usually unspecified internal "torque" of the form $\left\{\tau_{\text {int }}^{(0)}, 0,0,0\right\}$ that cancels any internal forces generated by the potential $\phi(\mathbf{q}) .{ }^{13}$ That is, the four-vector notation necessitates the formal introduction of internal or constraint forces. ${ }^{13}$ If the body is assumed to consist of discrete particles and introducing the center of mass 


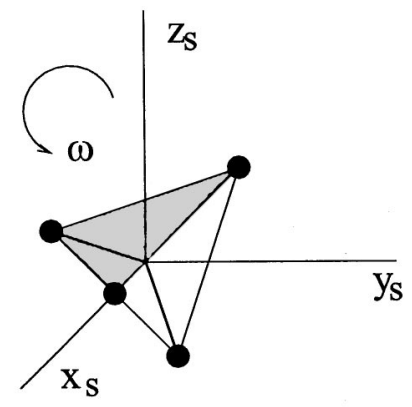

a

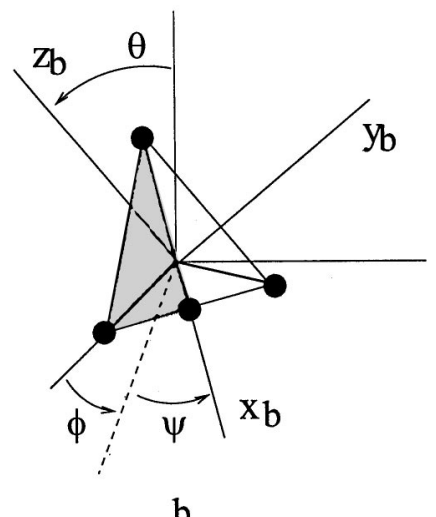

b

FIG. 1. (a) Angular velocity, $\omega$, rotates the body-fixed coordinate frame $\left(x_{b}, y_{b}, z_{b}\right)$ away from the system frame $\left(x_{s}, y_{s}, z_{s}\right)$. (b) Resulting rotation is specified by the Euler angles $(\phi, \theta, \psi)$. Angle $\phi$ is a counterclockwise rotation about the $z_{s}$ axis. This is followed by counterclockwise rotation of $\theta$ about the newly generated $x$ axis shown as a dotted line. Last, $\psi$ is a rotation about the newly generated $z$ axis.

$$
\mathbf{d}_{k}^{(s)}=\mathbf{A}^{T}(\mathbf{q}) \mathbf{d}_{k}^{(b)}, \quad \mathbf{d}_{k}^{(s)}=\mathbf{r}_{k}^{(s)}-\mathbf{r}_{\mathrm{cm}}^{(s)},
$$

with center of mass position $\mathbf{r}_{\mathrm{cm}}$. Then, it is easy to show that

$$
\begin{aligned}
\tau^{(4)} & =\left\{\sum_{k} \mathbf{F}_{k}^{(b)} \cdot \mathbf{d}_{k}^{(b)}, \sum_{k} \mathbf{d}_{k}^{(b)} \times \mathbf{F}_{k}^{(b)}\right\}+\tau_{\text {int }}^{(4)}, \\
\tau^{(4)} & =\left\{0, \tau_{x}, \tau_{y}, \tau_{z}\right\},
\end{aligned}
$$

where $\mathbf{F}_{k}$ is the external force on the $k$ th particle. Again, external forces acting to deform the body are exactly balanced by the typically unspecified internal/constraint forces contained in $\tau_{\text {int }}^{(0)}$ and the first element of $\tau^{(4)}$ vanishes, identically. ${ }^{13}$ (This explicit treatment of the internal forces is provided to remind nonexpert reader that standard biophysical force fields/potential functions do, indeed, contain forces acting to deform the body and internal forces/constraint forces must be invoked. ${ }^{13}$ ) The equations of motion conserve the sum of the rotational kinetic energy plus the potential energy,

$$
E=T(\omega)+\phi(\mathbf{q})=\frac{1}{2} I_{x x} \omega_{x}^{2}+\frac{1}{2} I_{y y} \omega_{y}^{2}+\frac{1}{2} I_{z z} \omega_{z}^{2}+\phi(\mathbf{q}),
$$

although they are not Hamiltonian.

Given the symmetry of the quaternion equations of motion, it is natural to postulate the extended Lagrangian, ${ }^{16}$

$$
L=T\left(\omega^{(4)}\right)-\phi(\mathbf{q}),
$$

with

$$
T\left(\omega^{(4)}\right)=T(\omega)+\frac{1}{2} I_{00} \omega_{0}^{2}
$$

by redefining

$$
\omega^{(4)}=2 \mathbf{S}^{T}(\mathbf{q}) \dot{\mathbf{q}}=\left\{\omega_{0}, \omega_{x}, \omega_{y}, \omega_{z}\right\} .
$$

A Legendre transform can then be performed to yield the extended Hamiltonian

$$
H(\mathbf{p}, \mathbf{q})=\frac{1}{8} \mathbf{p}^{T} \mathbf{S}(\mathbf{q}) \mathbf{D S}^{T}(\mathbf{q}) \mathbf{p}+\phi(\mathbf{q})=T(\mathbf{p}, \mathbf{q})+\phi(\mathbf{q}),
$$

where

$$
\begin{aligned}
& \mathbf{p}=\frac{2}{|q|^{4}} \mathbf{S}(\mathbf{q}) \mathbf{D}^{-1} \omega^{(4)} \\
& \mathbf{D}=\left(\begin{array}{cccc}
I_{00}^{-1} & 0 & 0 & 0 \\
0 & I_{x x}^{-1} & 0 & 0 \\
0 & 0 & I_{y y}^{-1} & 0 \\
0 & 0 & 0 & I_{z z}^{-1}
\end{array}\right) .
\end{aligned}
$$

Using Hamilton's equations in the extended phase space,

$$
\begin{aligned}
& \dot{\mathbf{q}}=\nabla_{p} H(\mathbf{p}, \mathbf{q}), \\
& \dot{\mathbf{p}}=-\nabla_{q} H(\mathbf{p}, \mathbf{q}),
\end{aligned}
$$

it can be shown that

$$
\begin{aligned}
& \dot{\omega}_{0}=\frac{\omega_{0}^{2}}{|q|^{2}}, \\
& \dot{\omega}_{x}=\frac{\omega_{0} \omega_{x}}{|q|^{2}}+\frac{\tau_{x}}{I_{x x}}+\frac{\left(I_{y y}-I_{z z}\right)}{I_{x x}} \omega_{y} \omega_{z}, \\
& \dot{\omega}_{y}=\frac{\omega_{0} \omega_{y}}{|q|^{2}}+\frac{\tau_{y}}{I_{y y}}+\frac{\left(I_{z z}-I_{x x}\right)}{I_{y y}} \omega_{z} \omega_{x}, \\
& \dot{\omega}_{z}=\frac{\omega_{0} \omega_{z}}{|q|^{2}}+\frac{\tau_{z}}{I_{z z}}+\frac{\left(I_{x x}-I_{y y}\right)}{I_{z z}} \omega_{x} \omega_{y} .
\end{aligned}
$$

Therefore, if $\omega_{0}(0)=0$ and $\Sigma_{i} q_{i}^{2}(0)=|q(0)|^{2}=1$, unit quaternion dynamics are produced for all time $t$. In a key step, the properties of quaternion algebra ${ }^{13}$ are used to decompose, exactly, the extended Hamiltonian, Eq. (2.14), into a sum over permutation matrices,

$$
\begin{aligned}
& H(\mathbf{p}, \mathbf{q})=\sum_{k=0}^{3} h_{k}(\mathbf{p}, \mathbf{q})+\phi(\mathbf{q}), \\
& h_{k}(\mathbf{p}, \mathbf{q})=\frac{1}{8 I_{k}}\left[\mathbf{p}^{T} \mathbf{P}_{k} \mathbf{q}\right]^{2}, \\
& \mathbf{P}_{0} \mathbf{q}=\left\{q_{0}, q_{1}, q_{2}, q_{3}\right\}, \quad \mathbf{P}_{1} \mathbf{q}=\left\{-q_{1}, q_{0}, q_{3},-q_{2}\right\},
\end{aligned}
$$$$
\mathbf{P}_{2} \mathbf{q}=\left\{-q_{2},-q_{3}, q_{0}, q_{1}\right\}, \quad \mathbf{P}_{3} \mathbf{q}=\left\{-q_{3}, q_{2},-q_{1}, q_{0}\right\},
$$
where $I_{0}=I_{00}, \quad I_{1}=I_{x x}, \quad I_{2}=I_{y y}, \quad I_{3}=I_{z z} \quad$ and $\left(\mathbf{P P}^{T}\right)_{\alpha \beta}$ $=\delta_{\alpha \beta}$. If $\omega_{0}(0)=0$, then $h_{0}(\mathbf{p}, \mathbf{q})=0$, and remains zero for all time $t$ because each of the other "sub-Hamiltonians," $h_{k}(\mathbf{p}, \mathbf{q}), k \neq 0$, preserves the norm $|q|^{2}$, and the torque on the faux angular velocity $\omega_{0}$ is by definition zero. Equation (2.18) is denoted NO_SQUISH. 
For linear molecules/bodies, one of the eigenvalues of the moment inertia vanishes or, equivalently, in the bodyfixed frame one of the diagonal elements of the tensor is zero. The resulting singularity can be removed analytically and a special set of equations of motion and a corresponding numerical integrator for linear bodies constructed. Alternatively, for this special case, the Lagrange multipliers required by the standard SHAKE/RATTLE method can be determined, analytically; SHAKE yields a quadratic equation and RATTLE a linear equation. Thus, the most convenient and simple reversible symplectic integrator for linear bodies is the SHAKE/RATTLE algorithm, in conjunction with the analytical solutions for multipliers. Again, SHAKE/RATTLE numerical integration is, for a general case, nonreversible only because the Lagrange multipliers required by SHAKE must be obtained using an iterative procedure which is, furthermore, typically, solved to a fairly low tolerance. RATTLE yields a set of linear equations which can be always solved analytically. Note, SHAKE/RATTLE is neither reversible nor symplectic unless iterated to complete convergence ${ }^{15}$ (which is impractical unless an analytical solution for the multipliers can be found). Finally, rigid body algorithms cannot be used to treat partially constrained systems, for example, constraining the $\mathrm{C}-\mathrm{H}$ bonds but not the $\mathrm{H}-\mathrm{C}-\mathrm{H}$ bond angles in a $\mathrm{CH}_{3}$ group.

\section{B. Conserved quantities in numerical integration}

Hamilton's equations cannot be solved, exactly, in general, and numerical integration schemes must be developed. Briefly, a numerical integrator or a "map," is a finite time step $\Delta t$ approximation to the true dynamical equations that is applied iterative to produce a discrete approximate trajectory, $\widetilde{x}_{k}(n \Delta t)=\widetilde{x}_{k}[\widetilde{\mathbf{x}}([n-1] \Delta t) ; \Delta t]$ where $n$ is an integer. For a Hamiltonian system, one takes $k=1,2 N$ so that $\left\{\widetilde{x}_{k}, \widetilde{x}_{k+N}\right\}$ $\equiv\left\{\widetilde{p}_{k}, \widetilde{q}_{k}\right\}$. The approximate trajectory will share some but not all of the properties of the exact trajectory, $x_{k}(t)$ $=x_{k}[\mathbf{x}(t-\tau) ; \tau]$. Consider a "reversible" integrator. The term, reversible indicates that the integrator possesses time reversal symmetry like the original equations. That is, a trajectory evolved backwards from the final state leads to the same initial state, $\widetilde{x}_{k}([n-1] \Delta t) \equiv \widetilde{x}_{k}[\widetilde{\mathbf{x}}(n \Delta t) ;-\Delta t]$. Next, consider a "symplectic" integrator. The term symplectic implies that the integrator (of a Hamiltonian system) can be derived from a time-step-dependent Hamiltonian different from the original, $\widetilde{H}(\mathbf{p}, \mathbf{q} ; \Delta t) \neq H(\mathbf{p}, \mathbf{q})$ (see below for a precise definition of the symplectic property). This Hamiltonian, $\widetilde{H}(\mathbf{p}, \mathbf{q} ; \Delta t)$, is then, of course, preserved along the trajectory generated by the integrator. If an integrator is to be reversible and symplectic, $\widetilde{H}(\mathbf{p}, \mathbf{q} ; \Delta t)$ must be an even function of $\Delta t$ or $\widetilde{H}(\mathbf{p}, \mathbf{q} ; \Delta t)=\widetilde{H}(\mathbf{p}, \mathbf{q} ;-\Delta t)$. Thus, a symplectic integrator is not necessarily time reversible [i.e., a well defined $\widetilde{H}(\mathbf{p}, \mathbf{q} ; \Delta t)$ need not be an even function of $\Delta t]$. Furthermore, since Hamiltonian systems obey Liouville's theorem, a symplectic integrator is phase space volume preserving. ${ }^{13}$ However, beyond one physical dimension, a system that is phase space volume conserving, need not be symplectic. ${ }^{13}$ Finally, a reversible integrator need not be symplectic nor phase space volume preserving. More details are given below along with a novel generalization to non-Hamiltonian systems.

One powerful method to develop numerical integrators with desirable properties is the Trotter-Suzuki evolution operator splitting technique..$^{3,14,17,18}$ Here, the method is analyzed in order to demonstrate, clearly, how the properties described above arise and how they can be controlled. In the next subsection, the Trotter-Suzuki approach is applied to the systems of interest in this paper. In order to proceed further, consider the closed set of equations,

$$
\dot{\mathbf{x}}(t)=\mathbf{G}(\mathbf{x}(t)), \quad \mathbf{x}(t)=e^{i \mathcal{L} t} \mathbf{x}, \quad i \mathcal{L}=\mathbf{G}(\mathbf{x}) \cdot \nabla_{x},
$$

where the time evolution of the system has been expressed formally using the Liouville operator formalism and $\mathbf{x}(t)$ is the exact solution. It is often possible to decompose the full Liouville operator into a few simple parts, $i \mathcal{L}=i \mathcal{L}_{1}+i \mathcal{L}_{2}$, which can be applied analytically to yield a short time approximation to the true dynamics,

$$
\begin{aligned}
& e^{i \mathcal{L} t}=\prod_{k=1}^{P} e^{i \mathcal{L} \Delta t}, \quad e^{i \tilde{\mathcal{L}} \Delta t} \equiv e^{i \mathcal{L}_{1}(\Delta t / 2)} e^{i \mathcal{L}_{2} \Delta t} e^{i \mathcal{L}_{1}(\Delta t / 2)}, \\
& e^{i \mathcal{L} t}=\prod_{k=1}^{P} e^{i \tilde{\mathcal{L}} \Delta t}+\mathcal{O}\left(t \Delta t^{2}\right),
\end{aligned}
$$

where $\Delta t=t / P$ and the unitary property, $\exp (i \widetilde{\mathcal{L}} \Delta t)$ $\times \exp (-i \widetilde{\mathcal{L}} \Delta t)=1$, leads directly to time reversibility. Note, the error in the approximate evolution, in principle, increases linearly with time, $t$.

Fortunately, the Trotter-Suzuki approach allows a formal analysis that defines conditions under which more appealing error bounds exist. Applying the $\mathrm{BCH}$ (BakerCampbell-Hansdorf) formula ${ }^{14,19}$ to the integrator yields

$$
\begin{aligned}
& e^{i \tilde{\mathcal{L}} \Delta t}=e^{\Delta t}\left[i \mathcal{L}+\sum_{k=1}^{\infty} \Delta t^{2 k} C^{(k)}\right]=e^{\Delta t}\left[i \mathcal{L}+\sum_{k=1}^{\infty} \Delta t^{2 k} i \tilde{\mathcal{L}}^{(k)}\right], \\
& \prod_{k=1}^{P} e^{i \tilde{\mathcal{L}} \Delta t}=e^{0 P \Delta t}\left[i \mathcal{L}+\sum_{k=1}^{\infty} \Delta t^{2 k} \tilde{\mathcal{L}}^{(k)}\right]=e^{t}\left[i \mathcal{L}+\sum_{k=1}^{\infty} \Delta t^{2 k} i \tilde{\mathcal{L}}^{(k)}\right],
\end{aligned}
$$

because the commutator of any two Liouville operators yields a third,

$$
\widetilde{\mathcal{L}}^{(k)}=\widetilde{\mathbf{G}}^{(k)} \cdot \nabla_{x}
$$

and, for example,

$$
C^{(1)}=\frac{1}{24}\left(\mathcal{L}_{1}+2 \mathcal{L}_{2},\left[\mathcal{L}_{1}+\mathcal{L}_{2}\right]\right)=i \widetilde{\mathcal{L}}^{(1)} .
$$

Thus, the integrator generates the solution to the continuous time equations of motion,

$$
\dot{\tilde{x}}(t)=\sum_{k=0}^{\infty} \Delta t^{2 k} \widetilde{\mathbf{G}}^{(k)}(\widetilde{\mathbf{x}}(t)),
$$

at intervals $n \Delta t$, where $n$ is an integer, $\widetilde{\mathbf{G}}^{(0)}(\widetilde{\mathbf{x}}(t))$ $\equiv \mathbf{G}(\widetilde{\mathbf{x}}(t))$, and $\widetilde{\mathbf{x}}(t) \neq \mathbf{x}(t)$. In order to define error bounds, consider the case that the original equations possess a conserved quantity, 


$$
\frac{d H(\mathbf{x}(t))}{d t}=0
$$

or

$$
\mathbf{G}(\mathbf{x}) \cdot \nabla_{x} H(\mathbf{x})=0,
$$

and determine the conditions under which Eqs. (2.24) emit a corresponding conservation law, $\widetilde{H}(\widetilde{\mathbf{x}}(t) ; \Delta t)$ such that

$$
\widetilde{H}(\widetilde{\mathbf{x}}(t) ; \Delta t)=H(\widetilde{\mathbf{x}}(t))+\Delta t^{2} \widetilde{H}^{(1)}(\widetilde{\mathbf{x}}(t)),
$$

with no linear growth in time $t$. Formally expanding the proposed conserved quantity in a Maclaurin series with even powers of $\Delta t$ because the integrator is reversible,

$$
\widetilde{H}(\mathbf{x} ; \Delta t)=\sum_{k=0}^{\infty} \Delta t^{2 k} \widetilde{H}^{(k)}(\mathbf{x}),
$$

and setting the time derivative of $\widetilde{H}(\mathbf{x}(t) ; \Delta t)$ as defined by Eq. (2.24) equal to zero,

$$
\sum_{k=0}^{\infty} \Delta t^{2 k} \widetilde{\mathbf{G}}^{(k)}(\mathbf{x}) \cdot \nabla_{x} \widetilde{H}(\mathbf{x} ; \Delta t)=0,
$$

yields the recursion relation,

$$
\sum_{k=0}^{n} \widetilde{\mathbf{G}}^{(n-k)}(\mathbf{x}) \cdot \nabla_{x} \widetilde{H}^{(k)}(\mathbf{x})=0 .
$$

Equation (2.30) must be satisfied for all $n$ if $\widetilde{H}(\mathbf{x} ; \Delta t)$ is to exist. Note, the $n=0$ term vanishes identically because $H(\mathbf{x}) \equiv \widetilde{H}^{(0)}(\mathbf{x})$ and $\widetilde{\mathbf{G}}^{(0)}(\mathbf{x}) \equiv \mathbf{G}(\mathbf{x})$ and the integrator is, thus, second order. This expression, Eq. (2.30), for a general set of equations of motion has not appeared previously to our knowledge.

It is useful to see under what conditions, Eq. (2.30), can be made to vanish. If each of the "perturbation dynamics" $\widetilde{\mathbf{G}}^{(k)}(\mathbf{x})$ takes the form

$$
\widetilde{\mathbf{G}}^{(k)}(\mathbf{x})=\nabla_{x} \widetilde{H}^{(k)}(\mathbf{x}) \cdot \mathbf{g}^{-1}(\mathbf{x}),
$$

where $\mathbf{g}^{-1}(\mathbf{x})$ is an antisymmetric matrix with zero diagonal elements, then each perturbation dynamics has a conservation law $\widetilde{\mathbf{G}}^{(k)}(\mathbf{x}) \cdot \nabla_{x} \widetilde{H}^{(k)}(\mathbf{x}) \equiv 0$. Furthermore, Eq. (2.30) will be satisfied

$$
\sum_{k=0}^{n} \nabla_{x} \widetilde{H}^{(n-k)}(x) \cdot \mathbf{g}^{-1}(\mathbf{x}) \cdot \nabla_{x} \widetilde{H}^{(k)}(\mathbf{x})=0,
$$

by symmetry. Note, upon coordinate change $\mathbf{y}(\mathbf{x}), \mathbf{g}^{-1}(\mathbf{x})$ transforms like a metric tensor

$$
\begin{aligned}
\widetilde{\mathbf{G}}^{(k)}(\mathbf{y}) & =\nabla_{y} \widetilde{H}^{(k)}(\mathbf{y}) \cdot\left[\mathbf{J}(\mathbf{x}) \mathbf{g}^{-1}(\mathbf{x}) \mathbf{J}^{(T)}(\mathbf{x})\right] \\
& =\nabla_{y} \widetilde{H}^{(k)}(\mathbf{y}) \cdot \overline{\mathbf{g}}^{-1}(\mathbf{y}),
\end{aligned}
$$

where matrix $\mathbf{J}(\mathbf{x})=\partial \mathbf{y} / \partial \mathbf{x}$. If in any coordinate system $\mathbf{g}^{-1}(\mathbf{x})$ is an antisymmetric matrix with zero diagonal elements, it retains this form in all coordinate systems. Furthermore, if, in any coordinate system, $\mathbf{g}^{-1}$ can be transformed into a matrix of constants, then the phase space is flat ${ }^{20-22}$ and the determinant of the metric in this special coordinate system is defined to be unity, $\operatorname{det} \mathbf{g}^{-1} \equiv 1$, with the required constant absorbed into the conserved quantity/quantities. For this latter case, $\mathbf{g}^{-1}$ is the phase space metric tensor (see below). ${ }^{20-22}$

The above, general, analysis allows the choice of decomposition, $\mathcal{L}=\mathcal{L}_{1}+\mathcal{L}_{2}$ to be connected directly to properties of the integrator. If the original equations are Hamiltonian, then

$\mathbf{x}=\{\mathbf{p}, \mathbf{q}\}$,

$\mathbf{G}(\mathbf{x})=\nabla_{x} H(\mathbf{x}) \cdot \mathbf{g}^{-1}=\mathbf{G}(\mathbf{p}, \mathbf{q})=\left\{-\nabla_{q} H(\mathbf{p}, \mathbf{q}), \nabla_{p} H(\mathbf{p}, \mathbf{q})\right\}$,

$\mathbf{g}^{-1}=\left(\begin{array}{cc}0 & \mathbf{I} \\ -\mathbf{I} & 0\end{array}\right)$.

Following Ref. 5, if $i \mathcal{L}_{1}, i \mathcal{L}_{2}$ are each derivable from Hamiltonians,

$i \mathcal{L}_{1}=\nabla_{x} h_{1}(\mathbf{x}) \cdot \mathbf{g}^{-1}=\nabla_{p} h_{1}(\mathbf{p}, \mathbf{q}) \cdot \nabla_{q}-\nabla_{q} h_{1}(\mathbf{p}, \mathbf{q}) \cdot \nabla_{p}$,

$i \mathcal{L}_{2}=\nabla_{x} h_{2}(\mathbf{x}) \cdot \mathbf{g}^{-1}=\nabla_{p} h_{2}(\mathbf{p}, \mathbf{q}) \cdot \nabla_{q}-\nabla_{q} h_{2}(\mathbf{p}, \mathbf{q}) \cdot \nabla_{p}$,

$$
H(\mathbf{x})=h_{1}(\mathbf{x})+h_{2}(\mathbf{x})=H(\mathbf{p}, \mathbf{q})=h_{1}(\mathbf{p}, \mathbf{q})+h_{2}(\mathbf{p}, \mathbf{q}),
$$

then each $\widetilde{\mathbf{G}}^{(k)}(\mathbf{x})=\widetilde{\mathbf{G}}^{(k)}(\mathbf{p}, \mathbf{q})$ is Hamiltonian, i.e., derived from $\widetilde{H}^{(k)}(\mathbf{x})=\widetilde{H}^{(k)}(\mathbf{p}, \mathbf{q}), \widetilde{H}(\mathbf{p}, \mathbf{q} ; \Delta t)$ is the Hamiltonian of Eq. (2.24) and Eq. (2.30) is trivially satisfied. This occurs because the commutator of any two Hamiltonian Liouville operators yields a third, whose associated Hamiltonian is given by the Poisson bracket, $h_{3}=\left\{h_{1}, h_{2}\right\}$, i.e.,

$i \mathcal{L}_{3}=\left[i \mathcal{L}_{1}, i \mathcal{L}_{2}\right]$,

$i \mathcal{L}_{3}=\nabla_{x} h_{3}(\mathbf{x}) \cdot \mathbf{g}^{-1} \cdot \nabla_{x}=\nabla_{p} h_{3}(\mathbf{p}, \mathbf{q}) \cdot \nabla_{q}-\nabla_{q} h_{3}(\mathbf{p}, \mathbf{q}) \cdot \nabla_{p}$,

$$
\begin{aligned}
h_{3}(\mathbf{x}) & =\nabla_{x} h_{1}(\mathbf{x}) \cdot \mathbf{g}^{-1} \cdot \nabla_{x} h_{2}(\mathbf{x})=h_{3}(\mathbf{p}, \mathbf{q})=\left\{h_{1}, h_{2}\right\}, \\
& =\nabla_{p} h_{1}(\mathbf{p}, \mathbf{q}) \cdot \nabla_{q} h_{2}(\mathbf{p}, \mathbf{q})-\nabla_{q} h_{1}(\mathbf{p}, \mathbf{q}) \cdot \nabla_{p} h_{2}(\mathbf{p}, \mathbf{q}) .
\end{aligned}
$$

Thus, for decompositions of the form of Eq. (2.35), it is straightforward, if tedious, to construct all the coefficients of the Maclaurin series expansion of $\widetilde{H}(\mathbf{p}, \mathbf{q} ; \Delta t)$ from nested Poisson brackets and the $\mathrm{BCH}$ expansion. ${ }^{5,19}$ For example, given the symmetric decomposition of Eq. (2.20), it follows from the first term of the BCH expansion and Eq. (2.36) that

$$
\widetilde{H}^{(1)}(\mathbf{p}, \mathbf{q})=\frac{1}{24}\left\{h_{1}+2 h_{2},\left\{h_{1}+h_{2}\right\}\right\} .
$$

Note, if an asymmetric decomposition were taken,

$$
e^{i \tilde{\mathcal{L}} \Delta t} \equiv e^{i \mathcal{L}_{2} \Delta t} e^{i \mathcal{L}_{1} \Delta t}=e^{i \mathcal{L} \Delta t}+\mathcal{O}\left(\Delta t^{2}\right),
$$

as opposed to the second-order symmetric decomposition of Eq. (2.20) and $i \mathcal{L}_{1}, i \mathcal{L}_{2}$ were each derivable from Hamiltonians following Eq. (2.35), then the resulting first-order integrator would possess a conserved quantity but would not possess time reversal symmetry, $\widetilde{H}(\mathbf{p}, \mathbf{q} ; \Delta t) \neq \widetilde{H}(\mathbf{p}, \mathbf{q} ;-\Delta t)$ or $e^{i \tilde{\mathcal{L}} \Delta t} e^{-i \tilde{\mathcal{L}} \Delta t} \neq 1$ (see Ref. 5, Chap. 12).

The derivation described above is generalized from that given in Ref. 5. Here, it is clear from the presentation the key result, Eq. (2.36), holds for any antisymmetric matrix of constants with zero diagonal elements $\mathbf{g}^{-1}$, not just the form of 
Eq. (2.34). In addition, Eq. (2.36) holds, if a transformation to any coordinate system, $\mathbf{y}(\mathbf{x})$ where $\overline{\mathbf{g}}^{-1}(\mathbf{y})=\overline{\mathbf{g}}^{-1}$ is a constant antisymmetric matrix with zero diagonal elements exists,

$$
\begin{aligned}
{\left[i \mathcal{L}_{1}, i \mathcal{L}_{2}\right]=} & \left\{\left[\nabla_{x} h_{1}(\mathbf{x}) \cdot \mathbf{g}^{-1}(\mathbf{x}) \cdot \nabla_{x}\right],\left[\nabla_{x} h_{2}(\mathbf{x}) \cdot \mathbf{g}^{-1}(\mathbf{x}) \cdot \nabla_{x}\right]\right\} \\
= & \left\{\left[\nabla_{y} h_{1}(\mathbf{y}) \cdot \mathbf{J}(\mathbf{y}) \mathbf{g}^{-1}(\mathbf{x}) \mathbf{J}^{T}(\mathbf{y}) \cdot \nabla_{y}\right],\right. \\
& {\left.\left[\nabla_{y} h_{2}(\mathbf{y}) \cdot \mathbf{J}(\mathbf{y}) \mathbf{g}^{-1}(\mathbf{x}) \mathbf{J}^{T}(y) \cdot \nabla_{y}\right]\right\} } \\
= & \left\{\left[\nabla_{y} h_{1}(\mathbf{y}) \cdot \overline{\mathbf{g}}^{-1} \cdot \nabla_{y}\right],\left[\nabla_{y} h_{3}(\mathbf{y}) \cdot \overline{\mathbf{g}}^{-1} \cdot \nabla_{y}\right]\right\} \\
= & \nabla_{y} h_{3}(y) \cdot \overline{\mathbf{g}}^{-1} \cdot \nabla_{y} \\
= & \nabla_{y} h_{3}(\mathbf{y}) \cdot \mathbf{J}(\mathbf{y}) \mathbf{g}^{-1}(\mathbf{x}) \mathbf{J}^{T}(\mathbf{y}) \cdot \nabla_{y} \\
= & \nabla_{x} h_{3}(\mathbf{x}) \cdot \mathbf{g}^{-1}(\mathbf{x}) \cdot \nabla_{x}, \\
h_{3}(x)= & \nabla_{y} h_{1}(\mathbf{y}) \cdot \overline{\mathbf{g}}^{-1} \cdot \nabla_{y} h_{2}(\mathbf{y}) \\
= & \nabla_{y} h_{1}(\mathbf{y}) \cdot \mathbf{J}(\mathbf{y}) \mathbf{g}^{-1}(\mathbf{x}) \mathbf{J}^{T}(\mathbf{y}) \cdot \nabla_{y} h_{2}(\mathbf{y}) \\
= & \nabla_{x} h_{1}(\mathbf{x}) \cdot \mathbf{g}^{-1}(\mathbf{x}) \cdot \nabla_{x} h_{2}(\mathbf{x}) .
\end{aligned}
$$

That is, for a system of equations of motion with a flat phase space, an integrator can be formulated with a conserved quantity following the procedure given above in any coordinate system. Simply using Liouville operator decompositions derived by splitting the conserved quantity $H(\mathbf{x})$ in proper combination with the metric tensor of the coordinate system of interest will yield integrators with conserved quantities by construction. Note, satisfying the above condition, Eq. (2.39), is sufficient for phase space to be flat.

Next, consider a system of equations of motion that can be written in the form described above,

$$
\dot{\mathbf{x}}(t)=\mathbf{G}(\mathbf{x}(t))=\nabla_{x(t)} H(\mathbf{x}(t)) \cdot \mathbf{g}^{-1}(\mathbf{x}(t)),
$$

where $\mathbf{g}^{-1}$ is taken to be a constant antisymmetric that has zero diagonal elements and $H(\mathbf{x}(t))$ is preserved. In general, one can view the equations of motion as coordinate transformation from $\mathbf{x}(0) \rightarrow \mathbf{x}[\mathbf{x}(0) ; t]$ parametrized by $t .^{20-22}$ Thus, the time derivative of the coordinates at time $t$ can be expressed in terms of the time derivative of the equations at time zero. For the case in which phase space is flat, $\mathbf{g}^{-1}(\mathbf{x}(t))=\mathbf{g}^{-1}$,

$$
\begin{aligned}
\mathbf{G}(\mathbf{x}(0)) & =\nabla_{x(0)} H(\mathbf{x}(0)) \cdot\left[\mathbf{g}^{-1}\right], \\
\mathbf{G}(\mathbf{x}(t)) & =\nabla_{x(t)} H(\mathbf{x}(0)) \cdot\left[\mathbf{J}(t) \mathbf{g}^{-1} \mathbf{J}^{T}(t)\right] \\
& =\nabla_{x(t)} H(\mathbf{x}(t)) \cdot\left[\mathbf{J}(t) \mathbf{g}^{-1} \mathbf{J}^{T}(t)\right],
\end{aligned}
$$

where $\mathbf{J}(t)=\partial \mathbf{x}(t) / \partial \mathbf{x}(0)$ and $H[\mathbf{x}(t)] \equiv H[\mathbf{x}(0)]$. This naturally leads to

$$
\begin{aligned}
& \mathbf{J}(t) \mathbf{g}^{-1} \mathbf{J}^{T}(t)=\mathbf{g}^{-1}, \\
& \mathbf{J}(t) \mathbf{g}^{-1}[\mathbf{y}(0)] \mathbf{J}^{T}(t)=\mathbf{g}^{-1}[\mathbf{y}(t)],
\end{aligned}
$$

so that the Eq. (2.41) is preserved. Equation (2.42) demonstrates the $\mathbf{g}^{-1}$ role as a metric tensor of phase space and, also, holds in a general coordinate system, $\mathbf{y}(\mathbf{x})$. For Hamiltonian systems,

$$
\mathbf{J}(t)=\left(\begin{array}{ll}
\frac{\partial \mathbf{p}(t)}{\partial \mathbf{p}(0)} & \frac{\partial \mathbf{p}(t)}{\partial \mathbf{q}(0)} \\
\frac{\partial \mathbf{q}(t)}{\partial \mathbf{p}(0)} & \frac{\partial \mathbf{q}(t)}{\partial \mathbf{q}(0)}
\end{array}\right), \quad \mathbf{g}^{-1}=\left(\begin{array}{cc}
0 & \mathbf{I} \\
-\mathbf{I} & 0
\end{array}\right)
$$

Equation (2.42) is referred to as the symplectic property, i.e., when $\mathbf{g}^{-1}$ is of the form in Eq. (2.43). Satisfying Eq. (2.42) is sufficient for a set of equations to possess a conserved quantity given a flat phase space.

It should be noted that the symplectic property is often employed to analyze numerical integrators of Hamiltonian systems. It is straightforward to compute an integrator's Jacobian matrix $\mathbf{J}$ and implicitly determine if an integrate possess continuous time equations of Hamiltonian form and, hence, a conserved quantity. The Liouville operator formalism, from which the equations of motion given in Eq. (2.24) arise naturally, has important advantages as Jacobian matrices of complex integrators are tedious to obtain, analytically. Also, simply considering the Jacobian matrix, only, misses the detailed connection to the equations of motion and the construction of $\widetilde{H}(\Delta t)$ from Poisson brackets provided by the evolution operator formalism (see Ref. 5). For systems with a flat phase space, the Liouville operator construction is also possible as described above. Equation (2.42) can be employed to check integrators for conserved quantities when a $\mathbf{g}^{-1}(\mathbf{x})$ is known or can be inferred provided phase space is flat [the metric satisfies Eq. (2.39)].

Next, phase space volume preservation is considered. For systems with flat phase space, again, $\mathbf{g}^{-1}$ has constant elements and unit determinant. Thus, Eq. (2.42) implies phase space volume preservation,

$$
\operatorname{det} \mathbf{J}(t)=1 \text {, }
$$

or, equivalently,

$$
d \mathbf{x}(t)=[\operatorname{det} \mathbf{J}(t)] d \mathbf{x}(0)=d \mathbf{x}(0),
$$

where $\operatorname{det} \mathbf{J}(t)$ for the transformation. Phase space volume preservation also embodies Liouville's theorem,

$$
\begin{aligned}
& \frac{d[\operatorname{det} \mathbf{J}(t)]}{d t}=[\operatorname{det} \mathbf{J}(t)] \nabla_{x} \cdot \dot{\mathbf{x}}(t), \\
& \frac{d \log [\operatorname{det} \mathbf{J}(t)]}{d t}=\kappa(t)=\nabla_{x(t)} \nabla_{x(t)} H(\mathbf{x}(t)) \cdot \mathbf{g}^{-1}=0,
\end{aligned}
$$

where $\kappa=\nabla_{x} \cdot \dot{\mathbf{x}}$ is called the compressibility of the dynamical system. Interested readers are encouraged to see Refs. 23 and 24 for a simple derivation of Eq. (2.46) starting from the definition of $\mathbf{J}(t)=\partial \mathbf{x}(t) / \partial \mathbf{x}(0)$. Of course, a constant or unit Jacobian is a necessary but not sufficient condition for a flat phase space to exist. All elements of $\mathbf{g}^{-1}$ must be constant.

Now, consider a set of equations of motion that have a Jacobian without explicit time dependence. That is, viewing the equations of motion as a variable transformation, it is possible to write 


$$
\begin{aligned}
& d \mathbf{x}(t)=d \mathbf{x}(0)\{\operatorname{det} \mathbf{J}[\mathbf{x}(t)]\} \\
&=d \mathbf{x}(0) \sqrt{\frac{\operatorname{det} \mathbf{g}^{-1}(\mathbf{x}(t))}{\operatorname{det} \mathbf{g}^{-1}(\mathbf{x}(0))}} \\
&=d \mathbf{x}(0) \sqrt{\frac{\operatorname{det} \mathbf{g}(\mathbf{x}(0))}{\operatorname{det} \mathbf{g}(\mathbf{x}(t))}}, \\
& d \mathbf{x}(t) \sqrt{\operatorname{det} \mathbf{g}(\mathbf{x}(t))}=d \mathbf{x}(0) \sqrt{\operatorname{det} \mathbf{g}(\mathbf{x}(0))},
\end{aligned}
$$

through the use of Eq. (2.42), valid when phase space is flat. Equation (2.47) is generally valid. Equation (2.48), again, illustrates the $\mathbf{g}(\mathbf{x}(t))$ role as the metric tensor of the phase space. $^{20-22}$ Here, the equations of motion are compressible and do not obey Liouville's theorem or preserve the phase space volume $[d \mathbf{x}(0) \neq d \mathbf{x}(t)]$. Nevertheless, they do possess an analogous measure conservation law, Eq. (2.48). ${ }^{23,24}$ Note, the measure conservation law holds for a general set of equations of motion ${ }^{23,24}$ not only for flat phase spaces but the identification of the metric tensor is not obvious. Finally, measure conservation and a time step dependent conserved quantity together allow a well defined, if time step dependent, statistical mechanical ensemble to be elucidated. ${ }^{25}$

Next, consider an integrator derived from the Liouville operator formalism which preserves a time-step-independent measure, $\operatorname{det} \widetilde{\mathbf{J}}(t)=\operatorname{det} \widetilde{\mathbf{J}}(\widetilde{\mathbf{x}}(t))$,

$$
\frac{d \log \{\operatorname{det} \widetilde{\mathbf{J}}(\widetilde{\mathbf{x}}(t))\}}{d t}-\nabla_{x} \cdot \dot{\tilde{\mathbf{x}}}=0,
$$$$
\sum_{k} \Delta t^{2 k}\left\{\widetilde{\mathbf{G}}^{(k)}(\mathbf{x}) \cdot \nabla_{x} \log [\operatorname{det} \widetilde{\mathbf{J}}(\mathbf{x})]-\nabla_{x} \cdot \widetilde{\mathbf{G}}^{(k)}(\mathbf{x})\right\}=0,
$$

$\widetilde{\mathbf{G}}^{(k)}(\mathbf{x}) \cdot \nabla_{x} \log [\operatorname{det} \widetilde{\mathbf{J}}(\mathbf{x})]-\widetilde{\boldsymbol{\kappa}}^{(k)}(\mathbf{x})=0$,

where Eq. (2.24) has been employed. This condition is sufficient to guarantee that the integrator preserves the same measure as the original set of equations, $\operatorname{det} \widetilde{\mathbf{J}}(\mathbf{x}(t))$ $\equiv \operatorname{det} \mathbf{J}(\mathbf{x}(t))$, because $\widetilde{\mathbf{G}}^{(0)}(\mathbf{x}) \equiv \mathbf{G}(\mathbf{x})$. In addition, (1) a common transformation can be made to the coordinate system in which all the perturbations $k$ are incompressible, $\widetilde{\kappa}^{(k)}=0$, or all the equations cannot vanish simultaneously in Eq. (2.49); (2) since an incompressible dynamics may be Hamiltonian or possess a flat phase space, the common transformation may lead to a set of variables in which all perturbation terms are Hamiltonian or have a flat phase space. Therefore, a measure preserving the integrator of a nonHamiltonian set of equations can, in principle, exhibit long time stability via a conserved quantity of the form, $\widetilde{H}(\mathbf{x} ; \Delta t)$ but is not guaranteed to do so. Lack of measure conservation indicates that no such transformation can exist. Hence, measure conservation is a necessary but not sufficient condition for conserved quantities to exist.

Last, it is useful to observe that $\widetilde{H}(\mathbf{x} ; \Delta t)$ will, in general, only be close to $H(\mathbf{x} ; \Delta t)$ for small $\Delta t$. Thus, the trajectories of the numerical integrator will be meaningful approximations to the true trajectories only in this limit. However, the existence of the conserved quantity does guarantee that there is a bound on the deviation of the integrator's trajectory from exact. In addition, the integrator can possess closed orbits close to those of the true system (i.e., if they are present). As a specific example, consider a one-dimensional harmonic oscillator

$$
H(p, q)=\frac{p^{2}}{2 m}+\frac{m \omega^{2} q^{2}}{2},
$$

integrated using a simple Trotter-Suzuki decomposition of the form, $i \mathcal{L}_{1}=-m \omega^{2} q, i \mathcal{L}_{2}=p$, to yield the velocity Verlet algorithm, ${ }^{17,26}$ which is symplectic. The conserved quantity/ integrator Hamiltonian is

$$
\begin{aligned}
\tilde{H}(p, q ; \Delta t)= & \frac{p^{2}\left[1-\left(\frac{\omega \Delta t}{2}\right)^{2}\right]^{-1 / 2}}{2 m} \\
& \left.+\frac{m \omega^{2} q^{2}\left[1-\left(\frac{\omega \Delta t}{2}\right)^{2}\right]^{1 / 2}}{2}\right) \\
& \times \frac{\arccos \left(1-\frac{\omega^{2} \Delta t^{2}}{2}\right)}{|\omega \Delta t|} .
\end{aligned}
$$

The integrator has closed orbits for $\omega \Delta t / 2<1$ and yields a good approximation to the true trajectories if $\omega \Delta t \ll 1$ [i.e., $\left.\lim _{\omega \Delta t \rightarrow 0} \tilde{H}(p, q ; \Delta t)=H(p, q)\right]$.

In summary, the properties of numerical integrators derived from Trotter decompositions have been discussed, in detail. In particular, decompositions of equations of motion defined by a conserved quantity and metric tensor, Eq. (2.41), have been investigated, in detail. It has been shown that if a set of equations of motion possess a flat phase space, then Trotter decomposing the conserved quantity in any coordinate system, will yield an integrator that, itself, emits a conserved quantity. This generalizes the results of Ref. 5, which strictly treats the Hamiltonian case in canonical variables. In addition, a generalized symplectic property has been derived for systems with a flat phase space, Eq. (2.42), that can be employed to check if an integrator possesses a conserved quantity. Finally, phase space volume conservation has been shown to be a necessary but not sufficient condition for an integrator to possess a conserved quantity, as might, well, be expected.

\section{Numerical integration of rigid bodies}

It is, now, straightforward to design a symplectic integrator for NO_SQUISH, Eq. (2.18). This Hamiltonian [i.e., Eq. (2.18)], is decomposed into five sub-Hamiltonians,

$$
h_{k}(\mathbf{p}, \mathbf{q}), \quad k=0,4, \quad h_{4}(\mathbf{p}, \mathbf{q})=\phi(\mathbf{q}),
$$

and the explicit form for the first four $\left[h_{k}(\mathbf{p}, \mathbf{q}), k=0,3\right]$ are given in Eq. (2.18). The symplectic integrator scheme is naturally formed by

$$
\begin{aligned}
e^{i \tilde{\mathcal{L}} \Delta t}= & e^{i \mathcal{L}_{4}(\Delta t / 2)}\left[e^{i \mathcal{L}_{3}(\delta t / 2)}\right. \\
& \left.\times e^{i \mathcal{L}_{2}(\delta t / 2)} e^{i \mathcal{L}_{1} \delta t} e^{i \mathcal{L}_{2}(\delta t / 2)} e^{i \mathcal{L}_{3}(\delta t / 2)}\right]^{m_{\mathrm{rot}}} e^{i \mathcal{L}_{4}(\Delta t / 2)}, \\
i \mathcal{L}_{k}= & \nabla_{p} h_{k}(\mathbf{p}, \mathbf{q}) \cdot \nabla_{q}-\nabla_{q} h_{k}(\mathbf{p}, \mathbf{q}) \cdot \nabla_{p},
\end{aligned}
$$


where $\delta t=\Delta t / m_{\text {rot }}, \exp (i \widetilde{\mathcal{L}} \Delta t)=\exp (i \mathcal{L} \Delta t)+\mathcal{O}\left(\Delta t^{3}\right)$, each of the four evolution operators is, itself, Hamiltonian and can be applied analytically. The integer parameter $m_{\text {rot }}$ can be employed to increase the accuracy of the computationally inexpensive portion involving free rotation ${ }^{3}$ (that is, at little computational cost, the accuracy of this part of the approximate evolution operator can be improved). Note,

$$
h_{0}[\mathbf{p}(t), \mathbf{q}(t)] \equiv 0, \quad \exp \left[i \mathcal{L}_{0} t\right] \equiv 1,
$$

and the approximate evolution precisely conserve

$$
|q(t)|^{2} \equiv 1, \quad \omega_{0}(t) \equiv 0 .
$$

The action of the first three evolution operators (1)-(3), takes the simple form

$$
\begin{aligned}
& e^{i \mathcal{L}_{k} \delta t} \mathbf{q}=\cos \left(\zeta_{k} \delta t\right) \mathbf{q}+\sin \left(\zeta_{k} \delta t\right) \mathbf{P}_{k} \mathbf{q}, \\
& e^{i \mathcal{L}_{k} \delta t} \mathbf{p}=\cos \left(\zeta_{k} \delta t\right) \mathbf{p}+\sin \left(\zeta_{k} \delta t\right) \mathbf{P}_{k} \mathbf{p},
\end{aligned}
$$

where

$$
\zeta_{k}=\frac{1}{4 I_{k}} \mathbf{p}^{T} \mathbf{P}_{k} \mathbf{q},
$$

and the fourth evolution operator translates the momenta by the force

$$
e^{i \mathcal{L}_{4}(\Delta t / 2)} \mathbf{p}_{k}=\mathbf{p}_{k}+\frac{\Delta t}{2} \mathbf{F}^{(4)} .
$$

Of course, since $\phi(\mathbf{q})$, for realistic systems, contains internal forces, one must construct

$$
\tau_{\mathrm{ext}}^{(4)}=-\frac{1}{2} \mathbf{S}^{T}(\mathbf{q}) \nabla_{q} \phi(\mathbf{q}),
$$

generate $\tau^{(4)}$ from $\tau_{\text {ext }}^{(4)}$ by setting the first element of $\tau_{\text {ext }}^{(4)}$ to zero and solve for the forces ${ }^{13}$

$$
\mathbf{F}^{(4)}=2 \mathbf{S}(\mathbf{q}) \tau^{(4)} .
$$

Note, the Maclaurin series expansion of $\widetilde{H}(\mathbf{p}, \mathbf{q} ; \Delta t)$ for the NO_SQUISH integrator could be obtained using the analysis of the previous section. Also, the NO_SQUISH integrator could not be developed using a simple leap-frog or Taylor series approach but requires the Trotter-Suzuki technique. Some implementation details of the NO_SQUISH scheme are provided as a pseudocode in the Appendix. (See Refs. 3 and 8 for a pedagogical overview.)

In order to illustrate the properties of the Trotter-Suzuki operator factorization method on a non-Hamiltonian system, we decompose Eq. (2.5) into four parts following Ref. 12:

$i \mathcal{L}_{1}=\frac{1}{2}\left[\mathbf{S}(\mathbf{q}) \omega^{(4)}\right] \cdot \nabla_{q}$,

$i \mathcal{L}_{2}=\left[\frac{\left(I_{y y}-I_{z z}\right)}{I_{x x}} \omega_{y} \omega_{z}\right] \frac{\partial}{\omega_{x}}+\left[\frac{\left(I_{z z}-I_{y y}\right)}{I_{z z}} \omega_{y} \omega_{x}\right] \frac{\partial}{\omega_{z}}$,

$i \mathcal{L}_{3}=\left[\frac{\left(I_{z z}-I_{x x}\right)}{I_{y y}} \omega_{z} \omega_{x}\right] \frac{\partial}{\omega_{y}}+\left[\frac{\left(I_{x x}-I_{z z}\right)}{I_{z z}} \omega_{y} \omega_{x}\right] \frac{\partial}{\omega_{z}}$,

$i \mathcal{L}_{4}=\frac{\tau_{x}}{I_{x x}} \frac{\partial}{\omega_{x}}+\frac{\tau_{y}}{I_{y y}} \frac{\partial}{\omega_{y}}+\frac{\tau_{z}}{I_{z z}} \frac{\partial}{\omega_{z}}$,

and define an approximate evolution operator analogous to Eq. (2.53). Here, $i \mathcal{L}_{k}$ are not Hamiltonian, the evolution is not symplectic but phase space volume preserving, a special
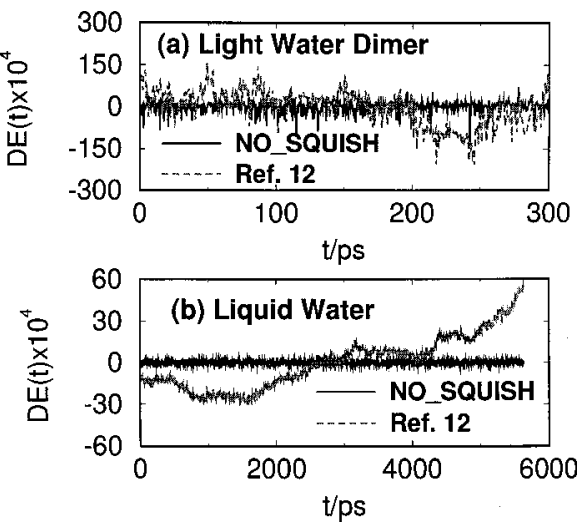

FIG. 2. (a) Light water dimer $\left(m_{H}=0.1 \mathrm{amu}\right)$ at $\langle T\rangle=155 \mathrm{~K}, \Delta t=0.3 \mathrm{fs}$. The energy conservation measure, $D E(t)=[E(t)-\bar{E}] /|\bar{E}|$, for NO_SQUISH (solid line) and Ref. 12 (dotted line). (b) Liquid water at $\langle T\rangle=295 \mathrm{~K}, \quad \rho=1 \mathrm{~g} / \mathrm{cm}^{3} \quad\left(m_{H}=1.008 \mathrm{amu}\right), \quad \Delta t=3.0 \mathrm{fs}$. NO_SQUISH (solid line) and Ref. 12 (dotted line).

case of measure conservation, that permits a conserved quantity, $\widetilde{H}(\mathbf{x} ; \Delta t)$, to exist but does not guarantee existence as described above. Note, if $\omega_{0}$ is introduced, explicitly, the equations of motion can be written in the form of Eq. (2.41) for which the phase space is flat (i.e., a variable transformation to a Hamiltonian system can been constructed; it is NO_SQUISH). Thus, a Lioville operator decomposition could be developed using $\left\{\omega^{(4)}, \mathbf{q}\right\}$ in conjunction with its metric tensor as described in Sec. II B. However, the decomposition of Ref. 12, Eq. (2.61), is not of this form.

\section{Rigid bodies in biophysical systems}

It is important to discuss the applicability of using rigid body algorithms to simulate biophysical systems. First, standard force fields treat the water molecules as rigid bodies and the methodology is, thus, perfectly adapted for these degrees of freedom. ${ }^{27}$ Second, in a peptide or protein it is useful to treat groups involving hydrogens, $\mathrm{CH}_{3}, \mathrm{CH}_{2}, \mathrm{NH}_{2}, \mathrm{OH}$, $\mathrm{NH}, \mathrm{CH}$, and $\mathrm{SH}$, as rigid moieties because $\mathrm{X}-\mathrm{H}$ bonds and $\mathrm{H}-\mathrm{X}-\mathrm{H}$ bonds have the highest frequencies in the molecule. (The lower-frequency CO motions are permitted to "flex.") The work presented in Refs. 28 and 29 clearly show that this approach yields accurate results. That is, physically important distribution functions are not altered by treating hydrogen-containing groups as rigid bodies.

\section{RESULTS}

The two rigid body methods were tested on three problems, the water dimer, liquid water and a helical peptide solvated in water (using $m_{\mathrm{rot}}=1$ ). The rigid water-water interaction potential employed was TIP3P and the peptidepeptide, peptide-water interactions were taken from the CHARMM22 force field. ${ }^{27}$

In Fig. 2(a), an energy conservation measure along a trajectory is presented for the water dimer. NO_SQUISH is more accurate than Ref. 12 but both methods are stable at long times. In Fig. 2(b), results are presented for liquid water 


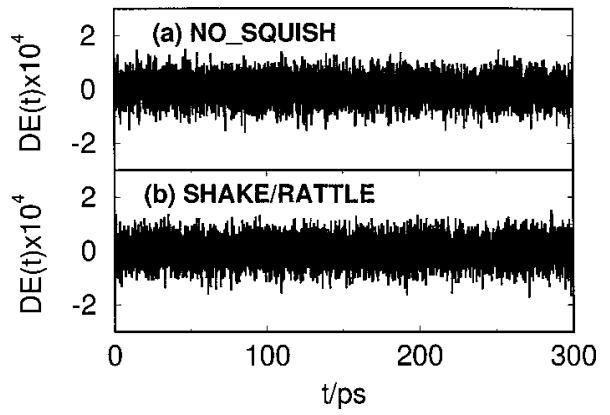

FIG. 3. Helical peptide in liquid water $\langle T\rangle=300 \mathrm{~K}, \Delta t=3.0$ fs. (a) Energy conservation measure for NO_SQUISH and (b) the SHAKE/RATTLE method (Refs. 6 and 7).

which exhibit similar trends. In general, the phase space volume preserving integrator performs well but not as well as the symplectic NO_SQUISH.

Next, a peptide (WEAQAREALAKEAARA) in water was studied using the PINY_MD simulation package ${ }^{30}$ with both the water molecules and all $\mathrm{CH}_{3}, \mathrm{CH}_{2}, \mathrm{NH}_{2}, \mathrm{OH}, \mathrm{NH}$, and $\mathrm{CH}$ groups in the peptide treated as rigid moieties while the $\mathrm{CO}$ groups were permitted to flex. Here, a comparison of NO_SQUISH to the SHAKE/RATTLE method ${ }^{6,7}$ demonstrates the excellent performance of the new, reversible, symplectic technique. Note, SHAKE/RATTLE is symplectic and reversible only if the Lagrange multipliers are solved to full convergence/determined exactly ${ }^{15}$ otherwise, SHAKE/ RATTLE is neither reversible nor symplectic. ${ }^{15}$ Nonetheless, NO_SQUISH and SHAKE/RATTLE perform well and the average energy conservation measure is $3.5 \times 10^{-5}$ for both methods (see Fig. 3). This indicates that for the peptide/water system the error in the two integrators is dominated by potential coupling, not by the treatment of the rotational motion.

In general, NO_SQUISH can be employed in conjunction with HMC calculations while SHAKE/RATTLE cannot. It is faster than our specialized implementation of SHAKE/ RATTLE for small rigid bodies and it is significantly easier to implement efficiently for large bodies. Briefly, in our specialized implementation of SHAKE for rigid bodies, we linearize the SHAKE equations for the multipliers, solve the coupled equations via LU (lower/upper) decomposition (for nonplanar structures), and directly for planar structures, and iterate until convergence of the nonlinear equations is achieved. For large nonplanar rigid molecules of greater than four atoms, linear constraints are used to "build" the additional atoms onto the rigid frame formed by four specially chosen basis atoms, a procedure that is not general and rather awkward to implement, in practice (see, for instance, Refs. 28 and 31 for discussions of SHAKE/RATTLE implementations). Finally, the standard Runge-Kutta or gear predictor corrector integrators originally proposed for use ${ }^{8,9}$ do not conserve $|q|^{2}$, and have been shown to exhibit poor energy conservation compared to Ref. 12 and other methods elsewhere. ${ }^{10-12}$

\section{CONCLUSION}

In response to the challenges inherent in utilizing novel massively parallel architectures for biophysical MD studies, the equations of motion governing coupled sets of rigid bodies have been reinvestigated. A rigid body Hamiltonian for the unit quaternion, the minimal nonsingular representation of rotation most commonly used in MD simulations, ${ }^{9}$ was derived along with a reversible, symplectic, integration scheme (NO_SQUISH). Tests on model and realistic systems indicate that NO_SQUISH is superior to nonsymplectic but area preserving methods, ${ }^{12}$ equal to the nonreversible, nonsymplectic (for practical implementations) and, hence, less versatile SHAKE/RATTLE technique, ${ }^{6,7}$ and far more accurate than standard gear predictor corrector or RungeKutta methods proposed for use with the unit quaternion 25 years ago. ${ }^{8,9}$ Finally, a new theoretical analysis has been presented that gives an explicit condition for an integrator to possess a conserved quantity, an explicit expression for the conserved quantity of a symplectic integrator, the latter following and in accord with Ref. 5, and extension of the explicit expression to general systems with flat phase space.

\section{ACKNOWLEDGMENTS}

This work was supported by grants PRF-32139-AC, NSF-CHE-9625015, NSF-EIA-0081307 (G.J.M.), and IBM (all authors). The authors would like to thank Professor B. B. Laird and Professor R. Skeel for helpful comments.

\section{APPENDIX: NO_SQUISH IMPLEMENTATION DETAILS}

It is simple to implement the NO_SQUISH method within a MD standard code using velocity Verlet to integrate degrees of freedom not involved in a rigid body. In this work, SHAKE/RATTLE is used to treat linear rigid bodies/ rods, only. The other rigid bodies are divided into groups determine by the number of atoms in the body (atms/body=natm_body). For example, if three groups are defined with natm_body[1] $=3$, natm_body[2] $=4$, natm_body[3] $=5$ then all $\mathrm{H}_{2} \mathrm{O}, \mathrm{CH}_{2}, \mathrm{NH}_{2}$, etc., moieties will be evolved together in the first group and so on. In the following $\{p, r\}$ denote Cartesian momenta and position, $\left\{p_{-}\right.$com, $r_{-}$com $\}$, denote center of mass momenta and position, $\left\{q, p_{-} q u a\right\}$ denote quaternion momenta and position, $d_{-}$body the position of an atom in the body fixed frame, $\mathrm{dt}$ the time step, $\Delta t, d$ ti the small step, $\Delta t / m_{\text {rot }}$, $A, A_{-}$transpose the matrices $\mathbf{A}(\mathbf{q}), \mathbf{A}^{T}(\mathbf{q}), \operatorname{dot} A_{-}$transpose the matrix $d \mathbf{A}^{T}(\mathbf{q}) / d t=\dot{\mathbf{A}}^{T}(\mathbf{p}, \mathbf{q}), S$ the matrix $\mathbf{S}(\mathbf{q}), P_{-} k$ the constant matrix, $\mathbf{P}_{k}, \tau 3$ the torques and $\tau 4$ the four component torque introduced in the text. All Cartesian and center of mass vectors have three components while the quaternion contains four. Finally, two arrays of indices, index_vv[] and index_rb[][] are employed that indirectly address the array of Cartesian momenta and positions.

routine integrate()

no_squish_evolve_0_to_dt2();

loop over particles not contained in a nonlinear rigid body $p[$ index_v $v[i \mathrm{~atm}]]+=0.5^{*} d t^{*} F[$ index_v $v[i \mathrm{~atm}]] ;$ 
$r[$ index_v $v[i$ atm $]]+=$ $\left(p[\text { index_v } v[i \mathrm{~atm}]]^{*} d t\right) / m[$ index_v $v[i \mathrm{~atm}]] ;$

end loop

shake();

get_forces();

no_squish_evolve_dt2_to_dt () ;

loop over particles not contained in a nonlinear rigid body $p[$ index_v $v[i \mathrm{~atm}]]+=0.5^{*} d t^{*} F[$ index_v $v[i \mathrm{~atm}]]$; end loop rattle();

end routine

routine no_squish_evolve_0_to_dt2()

loop over the rigid body groups

create_quaternion_and_com_forces(rigid[ $i$ group $]$ );

$p_{-}$qua $=\operatorname{rigid}[i$ group $] . p_{\text {_qua; }}$

$F_{\text {_qua }}=\operatorname{rigid}[i$ group $] . F_{\text {_qua; }}$

$r_{-}$com $=$rigid $[i$ group $] . r_{-}$com;

$p_{-} \mathrm{com}=\operatorname{rigid}[i \operatorname{group}] \cdot p_{-} \mathrm{com}$;

$F_{-}$com $=\operatorname{rigid}[i$ group $] . F_{-}$com;

loop over bodies in this group

$p_{-}$qua $[i b]+=F_{-}$qua $[i b]^{*} d t^{*} 0.5$;

$p_{-} \operatorname{com}[i b]+=F_{-} \operatorname{com}[i b]^{*} d t^{*} 0.5$;

$r_{-} \operatorname{com}[i b]+=\left(p_{-} \operatorname{com}[i b] * d t\right) / M_{-} \operatorname{com}[i b]$;

end loop over bodies in this group

no_squish_free_rotor(rigid[ $i$ group $]$ );

create_Cartesian_positions(rigid[igroup]);

end loop over the rigid body groups

end routine

routine no_squish_evolve_dt2_to_dt()

loop over the rigid body groups

create_quaternion_ and_com_forces(rigid[igroup]);

$p \_$qua $=\operatorname{rigid}[i$ group $] . p_{\text {_qua; }}$

$F_{\text {_qua }}=\operatorname{rigid}[i$ group $] . F_{-}$qua;

$p_{-} \operatorname{com}=\operatorname{rigid}[i$ group $] . p_{-}$com;

$F \_$com $=$rigid $[i$ group $] . F_{-}$com;

loop over bodies in this group

$p_{\text {qqua }}[i b]+=F_{\text {qua }}[i b]^{*} d t^{*} 0.5$;

$p_{-} \operatorname{com}[i b]+=F_{-} \operatorname{com}[i b]^{*} d t^{*} 0.5$;

end loop over bodies in this group

create_Cartesian_velocities(rigid[igroup]);

end loop over the rigid body groups

end routine

routine create_Cartesian_positions(rigid)

create_A_transpose_and_S(rigid);

loop over bodies in this group

loop over bodies in this group

$$
\begin{aligned}
r[\text { index_rb } r i b][i \mathrm{~atm}]] & =r_{-} \operatorname{com}[i b] \\
& +A_{-} \operatorname{transpose}[i b]^{*} d_{-} \operatorname{body}[i b][i \mathrm{~atm}]
\end{aligned}
$$

end loop over bodies in this group

end loop over number of atms/body in this group

end routine

routine create_Cartesian_velocities(rigid)

create_dot $A_{\text {_transpose(rigid); }}$ loop over number of atms/body in this group

loop over bodies in this group

$$
\begin{aligned}
& p\left[\text { index } \_r b[i b][i \mathrm{~atm}]\right]= m\left[\text { index } \_r b[i b][i \mathrm{~atm}]\right] \\
& \times\left(p_{-} \operatorname{com}[i b] / M_{-} \operatorname{com}[i b]\right. \\
&\left.+\operatorname{dot} A_{-} \operatorname{transpose}[i b]^{*} d_{-} \operatorname{body}[i b][i \mathrm{~atm}]\right) ;
\end{aligned}
$$

end loop over bodies in this group

end loop over number of atms/body in this group

end routine

routine create_quaternion_and_com_forces(rigid)

loop over number of atms/body in group

loop over bodies in group

$F \_$com $[i b]+=F[$ index_r $r[i b][i \mathrm{~atm}]] ;$

end loop over bodies in this group

end loop over number of atms/body in this group

loop over number of atms/body in this group

loop over bodies in this group

$$
\begin{aligned}
& F_{-} \text {space }=F[\text { index_rb }[i b][i \mathrm{~atm}]] \\
& -F_{-} \operatorname{com}[i b]^{*}\left(m\left[\text { index } \_r b[i b][i \mathrm{~atm}]\right] / M_{-} \operatorname{com}[i b]\right) \\
& F_{-} \text {body }=A[i b]^{*} F_{-} \text {space; } \\
& \tau 3[i b]+=d_{-} \text {body }[i b][i \mathrm{~atm}]^{*}(\text { cross_prod })^{*} F_{-} \text {body; }
\end{aligned}
$$

end loop over bodies in this group

end loop over number of atms/body in this group

loop over bodies in this group

$$
\begin{aligned}
& \tau 4=(0, \tau 3[i b]) ; \\
& F_{-} \text {qua }[i b]=2 S^{*} \tau 4 ;
\end{aligned}
$$

end loop over bodies in this group end routine

routine no_squish_free_rotor(rigid)

loop over $m_{\text {_ }}$ rot small time steps

no_squish_rotate $(3, d t i / 2)$;

no_squish_rotate(2,dti/2);

no_squish_rotate $(1, d t i)$;

no_squish_rotate( $2, d t i / 2)$;

no_squish_rotate( $3, d t i / 2)$;

end loop over $m_{-}$rot small time steps

end routine

routine no_squish_rotate $(k, d t)$

loop over bodies in this group

zeta_dt $=d t^{*}\left(p_{-}\right.$qua $\left.[i b]^{*} P_{-} k^{*} q[i b]\right) /\left(4 I_{-} k\right)$;

$q[i b]=\cos \left(\text { zeta }_{-} d t\right)^{*} q[i b]+\sin \left(\text { zeta }_{-} d t\right)^{*} P_{-} k^{*} q[i b]$

$$
\begin{array}{r}
p_{-} \text {qua }[i b]=\cos (\text { zeta_d } d)^{*} p_{-} \text {qua }[i b] \\
+\sin (\text { zeta_dt })^{*} P_{-} k^{*} p_{\text {qua }}[i b]
\end{array}
$$

end loop over bodies in this group

end routine

\footnotetext{
${ }^{1}$ E. Pennisi, Science 288, 939 (2000).

${ }^{2}$ F. Allen et al., IBM Syst. J. 40, 310 (2001).

${ }^{3}$ M. E. Tuckerman, G. J. Martyna, and B. J. Berne, J. Chem. Phys. 97, 1990 (1992).

${ }^{4}$ H. Grubmuller, H. Heller, A. Windemeth, and K. Schulten, Mol. Simul. 6, 121 (1991).

${ }^{5}$ M. P. Calvo and J. M. Sanz-Sarna, Numerical Hamiltonian Problems (Chapman and Hall, London, U.K., 1994).
} 
${ }^{6}$ J. P. Ryckaert, G. Ciccotti, and H. J. C. Berendsen, J. Comput. Phys. 23, 327 (1977).

${ }^{7}$ H. C. Andersen, J. Comput. Phys. 52, 24 (1983).

${ }^{8}$ D. J. Evans, Mol. Phys. 34, 317 (1977)

${ }^{9}$ M. P. Allen and D. J. Tildesley, Computer Simulation of Liquids (Oxford University Press, Oxford, U.K., 1989).

${ }^{10}$ A. Dullweber, B. Leimkuhler, and R. McLachlan, J. Chem. Phys. 107, 5840 (1997)

${ }^{11}$ A. Kol, B. Laird, and B. Leimkuhler, J. Chem. Phys. 107, 2580 (1997).

${ }^{12}$ N. Matubayasi and M. Nakahara, J. Chem. Phys. 110, 3291 (1999).

${ }^{13}$ H. Goldstein, Classical Mechanics (Addison-Wesley, Reading, MA, 1980).

${ }^{14}$ H. F. Trotter, Proc. Am. Math. Soc. 10, 545 (1959).

${ }^{15}$ B. Leimkuhler and R. D. Skeel, J. Comput. Phys. 112, 117 (1994).

${ }^{16}$ H. C. Andersen, J. Chem. Phys. 72, 2384 (1980).

${ }^{17}$ M. Creutz and A. Goksch, Phys. Rev. Lett. 63, 9 (1989).

${ }^{18}$ G. J. Martyna, M. E. Tuckerman, D. J. Tobias, and M. L. Klein, Mol. Phys. 87, 1117 (1996).

${ }^{19}$ R. M. Wilcox, J. Math. Phys. 8, 962 (1967).

${ }^{20}$ B. A. Dubrovin, A. T. Fomenko, and S. P. Novikov, Modern Geometry-
Methods and Applications Part I (Springer, New York, 1985).

${ }^{21}$ B. A. Dubrovin, A. T. Fomenko, and S. P. Novikov, Modern GeometryMethods and Applications Part II (Springer, New York, 1985).

${ }^{22}$ B. Schutz, Geometrical Methods of Mathematical Physics (Cambridge University Press, Cambridge, U.K., 1987).

${ }^{23}$ M. E. Tuckerman, C. J. Mundy, and G. J. Martyna, Europhys. Lett. 45, 149 (1999).

${ }^{24}$ M. E. Tuckerman, Y. Liu, G. Ciccotti, and G. J. Martyna, J. Chem. Phys. 115, 1678 (2001).

${ }^{25}$ M.E. Tuckerman and G.J. Martyna, J. Phys. Chem. B 104, 159 (2000).

${ }^{26}$ W. C. Swope, H. C. Andersen, P. H. Berens, and K. R. Wilson, J. Chem. Phys. 76, 637 (1982).

${ }^{27}$ A. MacKerell, Jr. et al., J. Phys. Chem. B 102, 3586 (1998).

${ }^{28}$ G. Ciccotti and J. P. Ryckaert, Comput. Phys. Rep. 3, 345 (1986).

${ }^{29}$ B. Chen, M. G. Martin, and J. I. Siepmann, J. Phys. Chem. B 102, 2578 (1998).

${ }^{30}$ M. E. Tuckerman, D. A. Yarne, S. O. Samuelson, A. L. Hughes, and G. J. Martyna, Comput. Phys. Commun. 128, 333 (2000).

${ }^{31}$ V. Krautler, W. F. Van Gunsteren, and P. H. Hunenberger, J. Comput. Chem. 22, 501 (2001) 